\title{
Computational Finite Element Software Assisted Development of a 3D Inductively Coupled Power Transfer System
}

\author{
Pratik Raval $^{1 *}$, Dariusz Kacprzak ${ }^{1}$, Aiguo $\mathrm{Hu}^{1}$ \\ ${ }^{1}$ Department of Electrical and Computer Engineering, \\ The University of Auckland, Auckland, New Zealand \\ *corresponding author, E-mail: prav010@aucklanduni.ac.nz
}

\begin{abstract}
To date inductively coupled power transfer (ICPT) systems have already found many practical applications including battery charging pads. In fact, current charging platforms tend to largely support only one- or two-dimensional planar movement in load. This paper proposes a new concept of extending the aspect ratios of the operating power transfer volume of ICPT systems to support arbitrary three dimensional load movements with respect to the primary coils. This is done by use of modern finite element method analysis software to propose the primary and secondary magnetic structures of such an ICPT system. Firstly, two primary magnetic structures are proposed based on contrasting modes of operation and different field directions. This includes a single-phase and multi-phase current model. Next, a secondary magnetic structure is customized to be compatible with both primary structures. The resulting system is shown to produce a $3 \mathrm{D}$ power transfer volume for battery cell charging applications.
\end{abstract}

\section{Introduction}

The technique of transmitting power across an air-gap without interconnecting wires is well established via the technique termed magnetic induction [1-2]. Inherently, this phenomenon removes the inconveniences caused from physical wires by providing power in hard to reach places where conventional direct electrical connections are inconvenient, hazardous, or impossible, lowers maintenance requirements as there is less wear and tear from wet, dirty, moisturised and hazardous environments, provides enhanced safety as it is free of sparking and can be used in potentially explosive atmospheres and supports freedom of mechanical movement of any load(s) as opposed to a localised load(s). Generally, this makes WPT systems reliable and robust. Systems utilising this technique are often referred to as inductively coupled power transfer (ICPT) systems. Such systems are becoming increasingly ubiquitous in industry. Some examples include; monorail systems [1], people mover or transportation applications such electric cars, trains and buses [3], biomedical implantation [4] and more recently low power consumer battery charging applications [5-6].
Regarding low power consumer battery charging applications, several platform charging systems have been developed [2] [4-7]. Current commercial systems include the Powermat, WildCharger and WiTricity chargers. These systems readily support two-dimensional (2D) planar load displacements. However, this is problematic for three-dimensional (3D) load displacements in a cage-like environment to support potential applications in sensors, telemetry devices, medical equipment, low power electronic devices and more particularly charging AA battery cells. To date, several approaches have been made to a support 3D power transfer volumes but further research is still required to develop customized magnetic structures.

One of the first approaches was made by Schuder [7]. In [7] a technique is proposed combining three distinct primary coils to generate three orthogonal magnetic field vectors. In this arrangement, all three coils are excited with AC current simultaneously. However, the generated field was not sufficiently uniform for 3D free positioning of any load particularly away from the coils. Furthermore, the use of a single pick-up does not permit power induction irrespective of power pick-up position and orientation througout the entire 3D power zone. Another arrangement is the Helmholtz pair [4]. In this arrangement, three distinct pairs of primary coil pairs are placed along three orthogonal axes. This design also does not produce a sufficiently uniform distribution of field in the entire 3D power zone. Particularly, in a 3D application as the seperation distance between the Helmholtz coils is increased the performance of the arrangement reduces drastically. In such arrangements, the sinusoidal primary currents may be applied either simultaneously or sequentially to the primary windings to take advantage of the pick-up position and orientation for maximum power transfer. However, the primary and secondary magnetic structures proposed to date are not customized for power transfer.

The process of customizing the magnetic structures of ICPT systems may be done through use of modern Finite Element Method (FEM) analysis software. Previously, such software has been used for design and magnetic frequency analysis of a novel $\mathrm{S}$ pick-up for a monorail ICPT system [8]. Furthermore, [9] has developed and tested various pick-up designs developed by use of similar software. The 
implementation of such FEM has been described in [10] as an essential part of the magnetic modeling for analysis and improving modern ICPT systems. Such FEM software has been used in [11] for complex magnetic frequency analysis including various high frequency effects such as the skin effect, proximity effect, eddy currents and various complex material properties. In summary, [8-11] suggests the accuracy of such simulation software results can fall within 5\% of experimentally verified results. This motivates the need to develop FEM assisted magnetic structures to support 3D aspect ratios of the power transfer volume and develop a unique battery charging system.

\subsection{Finite element formulation}

Mathematically, a conceptual model for the FEM may be derived in terms of the magnetic field intensity $H$ [12] for a triangular element with three nodes. An equation governing each element can be derived by application of the method of weighted residue using the Galerkin approach [12]. This approach essentially begins with the wave equation in terms of the magnetic field intensity $H$. The weighted residual for a single two dimensional element $e$, in medium of conductivity $\sigma$, permeability $\mu$, permittivity $\epsilon$, operating angular frequency $\omega$, with domain $d \Omega$ may then be written as in Equation 1 where $g$ is a non-zero constant.

$$
e=\frac{\partial}{\partial x}\left(\alpha \frac{\partial H}{\partial x}\right)+\frac{\partial}{\partial y}\left(\alpha \frac{\partial H}{\partial y}\right)+\beta H-g
$$

where $\alpha_{x}=\frac{1}{\mu}, \alpha_{y}=\frac{1}{\mu}$ and $\beta=-\left(\jmath \omega \sigma-\omega^{2} \epsilon\right)$

In order to minimize Equation 1, multiply by a weight function $w$, then integrate over element area enforcing the equation 0 .

$$
\iint_{\Omega} w[e] d x d y=0
$$

By applying an identity to Equation 2 is rewritten as in Equation 3.

$$
\begin{array}{r}
\iint_{\Omega}\left[\frac{\partial}{\partial x}\left(w \alpha_{x} \frac{\partial H}{\partial x}\right)+\frac{\partial}{\partial y}\left(w \alpha_{y} \frac{\partial H}{\partial y}\right)\right] d x d y- \\
\iint_{\Omega}\left[\left(\alpha_{x} \frac{\partial w}{\partial x} \frac{\partial H}{\partial x}\right)+\left(\alpha_{y} \frac{\partial w}{\partial y} \frac{\partial H}{\partial y}\right)\right] d x d y \\
\quad+\iint_{\Omega} \beta \omega H d x d y=\iint_{\Omega} \omega g d x d y
\end{array}
$$

Applying Green's theorem to the first integrating term of Equation 3 results in Equation 4 where the integration is performed over a closed loop path $\mathrm{d} \ell$.

$$
\oint_{\ell} w\left[\alpha_{x} \frac{\partial H}{\partial x} n_{x}+\alpha_{y} \frac{\partial H}{\partial y} n_{y}\right] d \ell
$$

where $n_{x}$ and $n_{y}$ are components of the unit vector normal to boundary of element. Upon substituting this term back into Equation 3 yields Equation 5.

$$
\begin{array}{r}
-\iint_{\Omega}\left[\alpha_{x} \frac{\partial w}{\partial x} \frac{\partial H}{\partial x}+\left[\alpha_{y} \frac{\partial w}{\partial y} \frac{\partial H}{\partial y}\right] d x d y+\right. \\
\iint_{\Omega} \beta \omega H d x d y=\iint_{\Omega} w g d x d y= \\
\oint_{\ell} w\left[\alpha_{x} \frac{\partial H}{\partial x} n_{x}+\alpha_{y} \frac{\partial H}{\partial y} n_{y}\right] d \ell
\end{array}
$$

Next, using a set of Lagrange polynomials to represent the magnetic field intensity as in Equation 6

$$
H=\sum_{j=0}^{n} H_{j}^{e} N_{j}
$$

where $N_{j}$ are the element shape functions based on the Lagrange polynomials and $n$ is the number of local nodes per element. Notably, the weight functions may also be written as a set of polynomials $w=N_{i}$, where $i=[1, n]$ so that Equation 5 is now written as Equation 7.

$$
\begin{array}{r}
-\iint_{\Omega}\left[\alpha_{x}\left(\frac{\partial N_{i}}{\partial x}\right)\left(\sum_{j=0}^{n} H_{j}^{e} \frac{\partial N_{j}}{\partial x}\right)+\right. \\
\left.\alpha_{y}\left(\frac{\partial N_{i}}{\partial y}\right)\left(\sum_{j=0}^{n} H_{j}^{e} \frac{\partial N_{j}}{\partial y}\right)\right] d x d y+ \\
\iint_{\Omega} \beta N_{i}\left(\sum_{j=0}^{n} H_{j}^{e} N_{j}\right) d x d y= \\
\iint_{\Omega} N_{i} g d x d y-\oint_{\ell} N_{i}\left(\alpha_{x} \frac{\partial H}{\partial x} n_{x}+\alpha_{y} \frac{\partial H}{\partial y} n_{y}\right) d \ell
\end{array}
$$

Equation 7 may be simplified and rewritten in matrix form. For a single two-dimensional triangular element in cartesian co-ordinates $(\mathrm{x}, \mathrm{y})$ with three nodes $(1,2,3)$ of area $\mathrm{A}$, the following compact matrix Equation 8 arises.

$$
[T+M] H=0
$$

where $T$ and $M$ are given by Equations 9 and 10 where $N_{i}$ is a $3 x 1$ matrix and $N_{j}$ is a $1 x 3$ matrix composed of the element shape functions integrated over element domain $\mathrm{d} \Omega$.

$$
\begin{array}{r}
T_{i j}^{e}=\beta \iint_{\Omega} N_{i} N_{j} d x d y=\frac{\beta A}{12}\left[\begin{array}{ccc}
2 & 1 & 1 \\
1 & 2 & 1 \\
1 & 1 & 2
\end{array}\right] \\
M=\frac{1}{\mu} \int_{\Omega}\left(\frac{\partial N_{i}}{\partial x} \frac{\partial N_{j}}{\partial x}+\frac{\partial N_{i}}{\partial y} \frac{\partial N_{j}}{\partial y}\right) d \Omega \\
=\alpha\left[\begin{array}{ccc}
M_{11} & M_{12} & M_{13} \\
& M_{22} & M_{23} \\
S y m & & M_{33}
\end{array}\right]
\end{array}
$$

where $\alpha=\frac{1}{4 \mu A}, M_{11}=y_{23}^{\overline{2}}+x_{32}^{\overline{2}}, M_{12}=\overline{y_{23}} \overline{y_{31}}+x_{32} \bar{x}_{13}^{-}$, $M_{13}=\overline{y_{23}} \overline{y_{12}}+\overline{x_{32}} \overline{x_{21}}, M_{22}=\bar{y}_{31} \bar{x} \overline{x_{13}^{2}}, M_{23}=\overline{y_{31}} \overline{y_{12}}+$ 
$x_{13}^{-} x_{21}$ and $M_{33}=y_{12}^{\overline{2}}+x_{21}^{\overline{2}}$, where $\overline{y_{i j}}=y_{i}-y_{j}$ or $\overline{x_{i j}}=$ $x_{i}-x_{j}$ for $i=[1,3]$ and $j=[1,3]$.

The result is that a single triangular element with 3 nodes, yields a $3 \times 3$ matrix. So, the ICPT system model with $n$ nodes will yield an $n x n$ matrix. Therefore, in order to automate this process, powerful FEM simulation software is used for magnetic frequency analysis at $155 \mathrm{kHz}$ and transient analysis at $6.45 \mu \mathrm{s}$.

\subsection{Proposed ICPT system}

The block diagram of the proposed ICPT system is shown in Figure 1. The input to the system is a DC voltage to the power converter for inversion into high frequency resonant AC current @ 155khz. The resonance is often required to lower the input VAR requirement. This stage is often termed primary compensation [13]. The outpuot current is fed to the primary windings that may typically wound around ferromagnetic material. The secondary magnetic structure intercepts the time varying flux for voltage induction into the secondary coils. This voltage is weak and so it is often resonanted. This stage is often termed secondary compensation [13]. Furthermore, the induced voltage may be of low quality and so power contioning may be required. Finally, the induced voltage is AC so it is rectified for satisfactory application to a load.

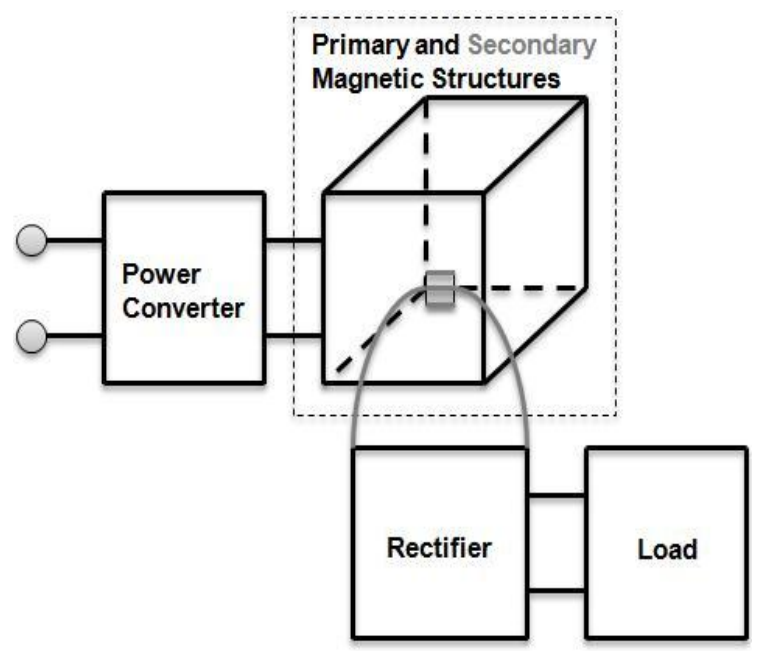

Figure 1: Structure of the proposed ICPT system.

\section{Development of primary magnetic structures}

The aim of the primary magnetic structure is to provide a satisfactory magnetic flux density (MFD) distribution in terms of magnitude and uniformity in a 3D intended power zone whilst minimizing the flux leakage to reduce potentially harmful electromagnetic interference (EMI). To do this, two magnetic structures with different coil orientation are proposed. Before presenting the structures, a methodology used for comparison is summarized in Equation 11. This method ensures the same magnetomotive force $(\mathrm{MMF})$ in ampere current-turns $(N I)$ ratio is used to account for the section length of coils $i$ in one model and a differing section length of coil $j$ of another model both of the same cross sectional area. This is equivalent to rewriting in terms of the the excitation input parameter of the current density $J$ of the FEM model as shown below.

$$
\sum_{1}^{i}(N I)_{i} l_{i}=\sum_{1}^{j}(N I)_{j} l_{j} \Leftrightarrow \sum_{1}^{i} J_{i} l_{i}=\sum_{1}^{j} J_{j} l_{j}
$$

For a rectangular winding structure, at larger distances it appears as a magnetic dipole and the magnetic field strength decays as $\frac{1}{z^{3}}$, where $z$ represents the vertical distance from the winding arrangement. Such characteristic results in a field that is weak due to a rapid decay within the middle region of the intended power zone. This motivates two models, a vertical field box and a horizontal field box. This is to spacially direct the resulting flux in orthogonal directions and contrast the effectiveness of the ferromagnetic casing for EMI shielding as will be evident in the following subsections.

Fundamentally, an arbitrarily oriented primary winding structure will generate each of the three required magnetic field vectors $B_{x}, B_{y}$ and $B_{z}$ in the space domain. Inherently, particular orientations in the winding structures will result in a dominant normal component $\left(B_{z}\right.$ or vertical) or tangential component $\left(B_{x}, B_{y}\right.$ or horizontal) in the magnitude of the magnetic flux density. In addition to this, the excitation of the winding structures in terms of the polarity of currents and phase-quadrature relationships may be developed as part of the magnetic circuit. Such design characteristics and parameters may be used advantageously to satisfy the requirement of generating a relatively uniform magnetic flux distribution within the three-dimensional powering volume. The entire intended powering volume is defined to be $190 \mathrm{~mm} \times 150 \mathrm{~mm} \times 100 \mathrm{~mm}$. As a result, the primary coils are of rectangular structure. The following sections presents the development of two models. The first is a single-phase current system model that is based on the predominant normal or vertical component defined as the magnitude component of $\left(B_{n}=\left|B_{z}\right|\right)$. The second is a multi-phase current system model that is based on the predominant tangential components defined as the magnitude of components $\left(B_{t}=\sqrt{B_{x}^{2}+B_{y}^{2}}\right)$.

\subsection{Single-phase model}

Firstly, a concept that is based on the production of a net vertical field is proposed as shown in Figure 2. The concept consists of overlayed winding structures with increasing coil turn density in the central region producing a net vertical field. The intention of this design is to compensate the central valley region with an additional peak provided by the additional coil turns in the middle layer with respect to the top and bottom-most layers. This is to provide a uniform as well as a strong magnitude component in magnetic flux density throughout the entire 3D power zone. Notably, 
in this model the primary windings are excited simulataneously and in-phase along as axes. The resulting magnitude of the magnetic field intensity vector $|H|$ is proportional to a single sinusoidal term [14] as in Equation 12

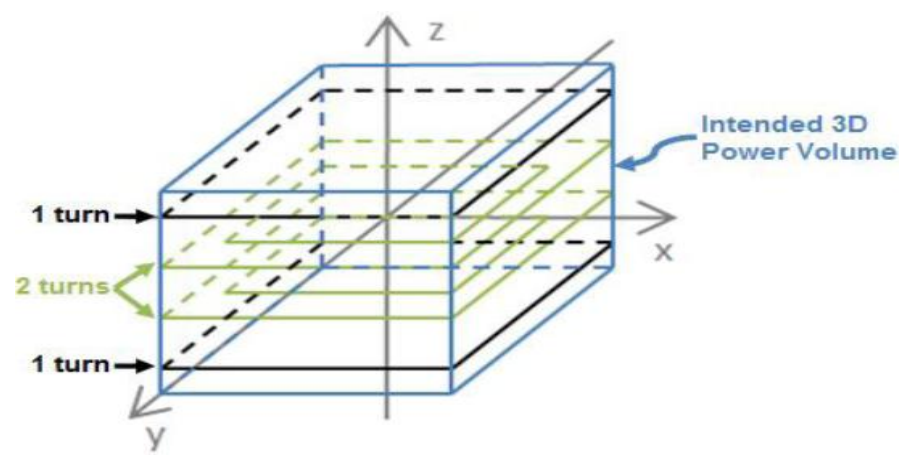

Figure 2: Vertical field conceptual box.

$$
|H| \propto \sqrt{3} I_{0} \sin \omega_{c} t+\phi
$$

where $I_{0}$ is the current amplitude, $\omega_{c}$ is the angular frequency and $\phi$ is the current phase angle.

The corresponding FEM model and simulation is shown in Figure 3. The concept is verified by the simulated MFD contour plot visualizing the formation of an additional peak in the absolute center of the power zone.
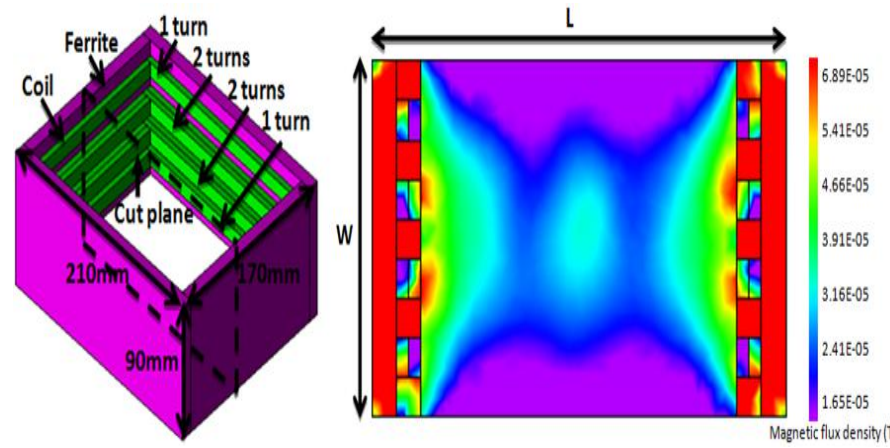

Figure 3: Vertical field FEM Model (left) and MFD distribution (right).

In this model, the currents are applied simultaneously and in-phase. Meanwhile, the dominance of the normal component of magnetic flux accounts for up to $86 \%$ to $98 \%$ of the total magnetic flux in the power zone. This data is extracted and visually evident from the magnetic flux density vector plot shown in Figure 4(a). This result shows the field largely vertically flowing during the entire cycle. The corresponding averaged MFD throughout the entire cubic power zone is $4.75 \mathrm{e}-05 \mathrm{~T}$. The MFD distribution may further be visualized through the cut-plane by extracting MFD values from the air-region mesh in $5 \mathrm{~mm}$ steps as plotted in Figure 4(b).

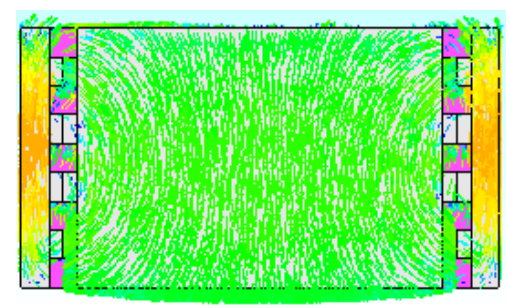

(a) MFD vectors.

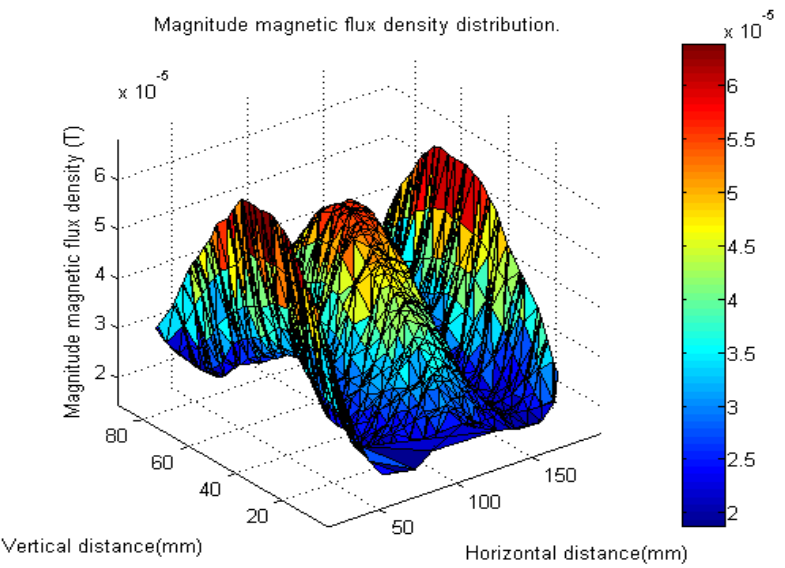

(b) MFD distribution.

Figure 4: Vertical field box MFD distribution.

\subsection{Multi-phase model}

Secondly, in an effort to lower the unintended radiation in the outer top and bottom-most regions, a horizontal field concept is proposed. In this concept, the horizontally oriented planar components of the magnetic flux density $B_{x}$, and $B_{y}$ that lie within a horizontal plane is intuitively made predominant in this concept. This is done by orienting four rectangular windings faced opposite and adjacent to each other to form a three-dimensional power emission volume as shown in Figure 5. This configuration is unique in that by utilising two planar components enables the sequential and phase quadrature application of the input primary currents. That is, the current magnitude of the current in each of the 4 rectangular windings is the same $\left|I_{1}\right|=\left|I_{2}\right|=$ $\left|I_{3}\right|=\left|I_{4}\right|$ but the current polarity of each winding opposes the directly opposite spiral such that $I_{1}(t)=-I_{2}(t)$ and $I_{1}\left(t-90^{\circ}\right)=I_{2}\left(t-90^{\circ}\right)$. Notably, the currents in two of the adjacently placed coils encounter a current in-phase quadrature with respect to the other two coils. This produces $B_{x}(t)$, while two of these spirals along the same plane produce a magnetic flux that is 90 degrees out of phase producing $B_{y}\left(t-90^{\circ}\right)$. This essentially produces a rotating magnetic field tracing an elliptical pathway as illustrated in Figure 5. For such a design, where the pri- 
mary currents must be applied sequentially and in phasequadrature, the resulting magnitude of the magnetic field intensity vector may be derived to show it is proportional to two time-variant terms to represent an elliptically rotating field vector [14] as in Equation 13.

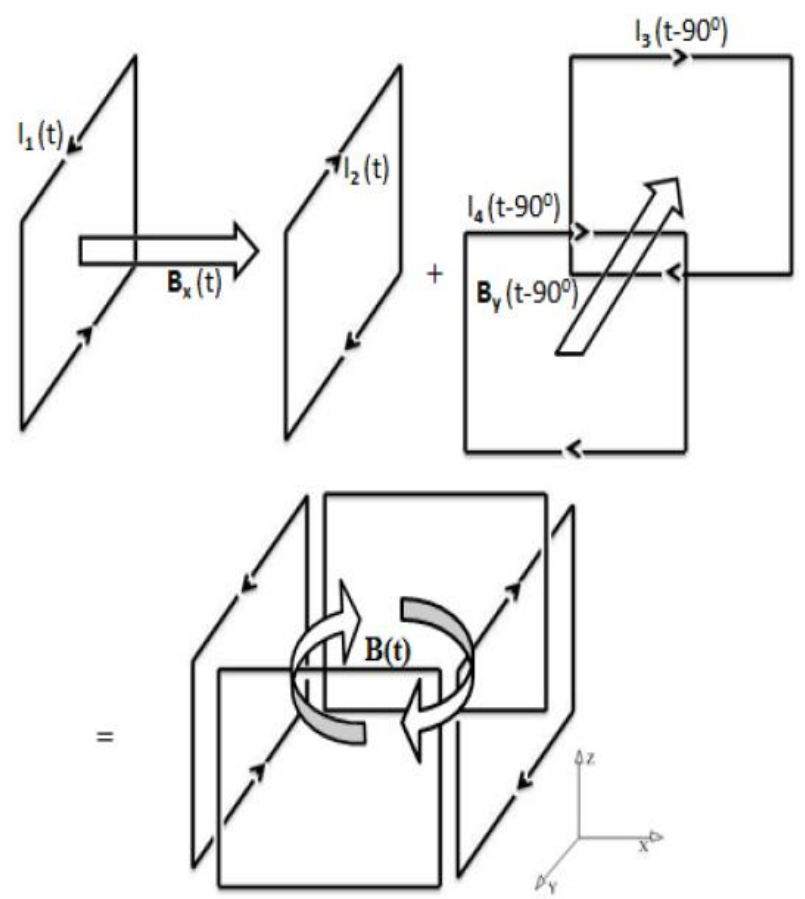

Figure 5: Horizontal field conceptual box.

$$
|H| \propto I_{0} \sqrt{\sum_{i=1}^{3}\left[\sin \left(\omega_{c} t\right) \cos \left(\phi_{i}\right)+\cos \left(\omega_{c} t\right) \sin \left(\phi_{i}\right)\right]^{2}}
$$

where the symbols have their usual meanings.

The horizontal field box is shown in Figure 6. In this box, two pairs of rectangular coils are aligned perpendicular to each other producing a net horizontal field. The exciting current is such that one pair of coils encounters a 90 degrees phase shift in current with respect to the other pair. The result is a rotating magnetic field. Instantaneously, only one pair of coils are switched on. The result is compared when two coils are fully switched on to reflect the scenario that occurs every cycle. This is justified as the averaged MFD throughout the cubic volume with both coil pairs fully switched on is $2.27 \mathrm{e}-05 \mathrm{~T}$ as opposed to the case of one coil pair switched on is $1.74 \mathrm{e}-05 \mathrm{~T}$. Hence, the increase in MFD with the second pair switched on is not very beneficial due to destructive interfernce by adjacent like poles. The corresponding MFD distribution is shown to produce a central valley region.

The field is relatively uniform between horizontal distances of $70-130 \mathrm{~mm}$ with a variation of $18.15 \%$. The tangential component in this box accounts for up to $76 \%$ to $91 \%$ of the total magnetic flux in the power zone. This data is extracted and visually evident from the magnetic
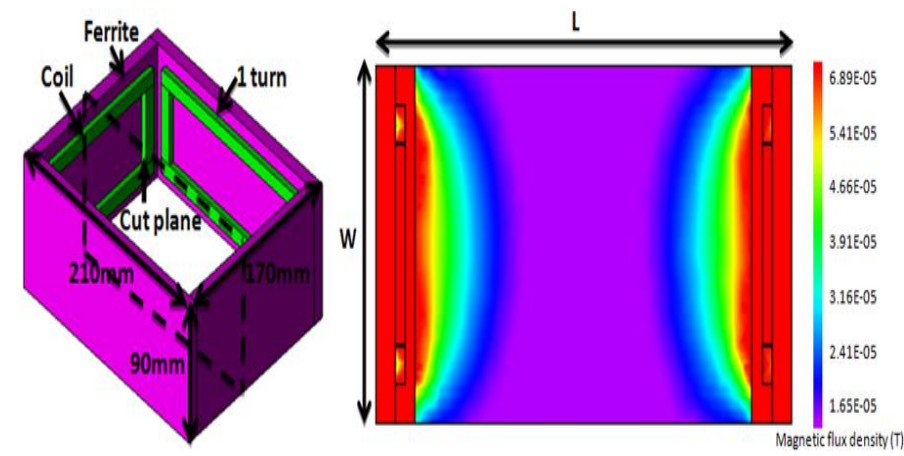

Figure 6: Horizontal field FEM Model (left) and MFD distribution (right).

flux density vector plot shown in Figure 7(a). This result shows the field largely horizontally flowing field during each half cycle. During the other half of the cycle, the adjacently placed coils will switch with a similarly horizontal flow of field. This provides information for pick-up design. The corresponding MFD distribution through the cut-plane is by extracting MFD values from the air-region mesh in $5 \mathrm{~mm}$ steps as plotted in Figure 7(b).

\section{Development of secondary magnetic structure}

The proposed secondary magnetic structure, as customized around the shape of the chargeable battery, is shown in Figure 8 . The pick-up was designed and customized to be compatible with both boxes. The objective of this pick-up is to induce power irrespective of pick-up position and orientation for charging a AA battery acting as a load. This is done by customizing a ferromagnetic core designed to induce the magnetic flux from three orthogonal axes. The design consists of a ferromagnetic cylindrical core surrounded by rectangular blocks of ferromagnets. The top view of the core shows the planar components of magnetic flux $B_{x}$ and $B_{y}$ being traversed through the core inducing a voltage along a single pick-up coil. The vertical component of magnetic flux $B_{z}$ is induced through the vertical crosssection of the cylindrical core as seen in the side core view. By including the tri-directional coils (x), (y) and (z) the resulting pick-up is also seen in Figure 8. Coils (x) and (y) as labeled account for the planar components of magnetic This requires a total of three series pick-up coils to account for the planar and normal components of induction. The battery may be accomodated within the air gap of the inner core.

For the purposes of the FEM simulation, the magnitude of the uncompensated power in each coil is summed as pick-ups in series. Notably, the uncompensated power $S_{u}$ is defined as the product between the open circuit voltage $V_{o c}$ and short circuit current $I_{s c}$ as shown in Equation 14. This is essentially the power level induced in the seconday pick-up coil before the resonant or compensation stage. Typically, the uncompensated power may be improved by a 


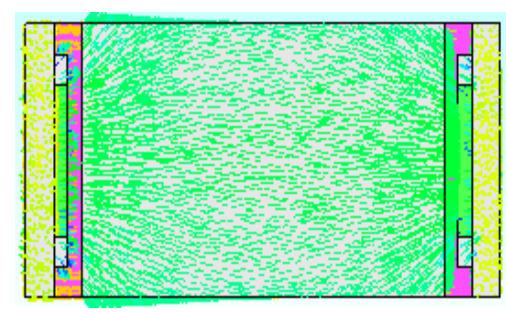

(a) MFD vectors.

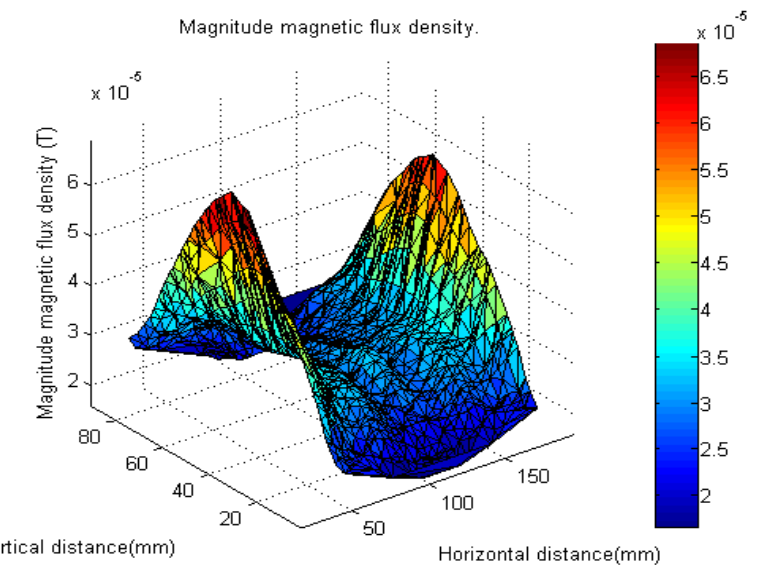

(b) MFD distribution.

Figure 7: Horizontal field box MFD distribution.

factor of the quality $Q$ of the compensation.

$$
S_{u}=V_{o c} \times I_{s c}
$$

The vertical orientation of the pick-up is tested through the center plane in $10 \mathrm{~mm}$ steps in both models as shown in Figure 9. The result shows an average total uncompensated power level of $1.4 \mathrm{~W}$ and $650 \mathrm{~mW}$ with a deviation of $101.3 \%$ for the vertical field box and and $150.08 \%$ for the horizontal field box.

Similarly, the horizontal orientation of the pick-up is tested through the center plane in $10 \mathrm{~mm}$ steps in both models as shown in Figure 9. The result shows an average total uncompensated power level of $1.07 \mathrm{~W}$ and $458 \mathrm{~mW}$ with deviation of $171.14 \%$ for the vertical field box and $188.25 \%$ for the horizontal field box.

\section{Conclusions}

This paper has demonstrated the concept of a threedimensional power transfer volume for a battery cell charging application by use of modern FEM assisted software to custom develop the magnetic structures of an ICPT system. This includes two primary structures based on contrasting field directions shown to produce a 3D power transfer volume. In addition, a secondary pick-up structure was pro-
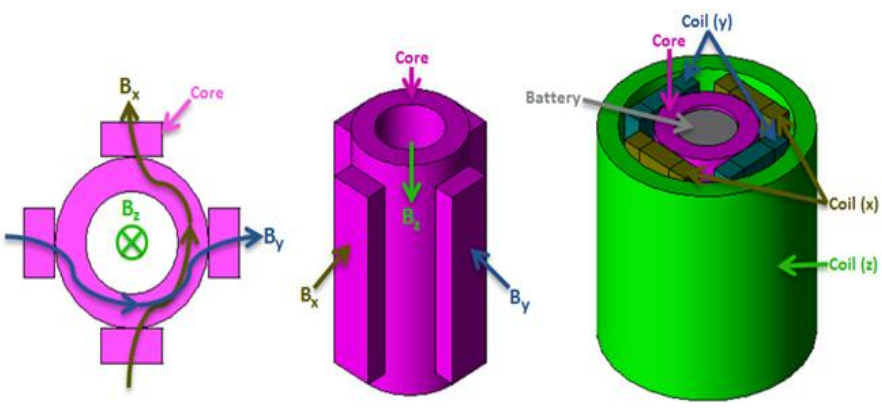

Figure 8: Proposed pick-up structure.

posed to accommodate battery cell charging and shown to induce three orthogonal field components.

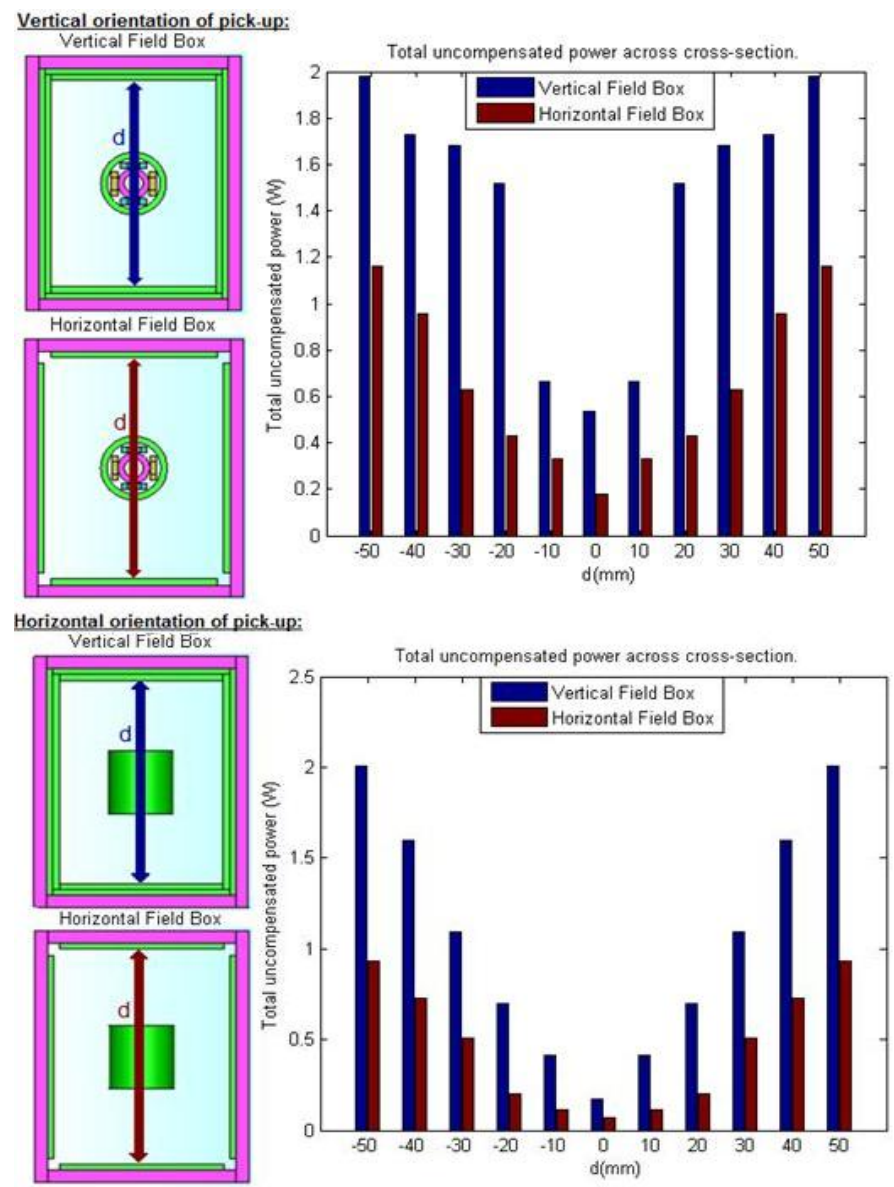

Figure 9: Pick-up performance. 


\section{References}

[1] A.W. Green, and J.T. Boys, $10 \mathrm{kHz}$ inductively coupled power transfer concept and control, IEEE PEVD conference, London, pp694-699, 1994.

[2] E. Abel, S. Third, "Contactless power transfer-An exercise in topology", IEEE Transactions on Magnetics, vol. 20, Issue: 5, pp. 1813-1815, 1984.

[3] G.A Covic, G.A. Elliott, O.H. Stielau, and R. M. Green, "The design of contactless energy transfer system for a people mover system", Proceedings of International conference on power system technology, Perth, Australia, pp79-94, December 2000.

[4] P. Si, Wireless power supply for implantable biomedical devices, $\mathrm{PhD}$ thesis, Deparment of Electrical and Computer Engineering, University of Auckland, 2008 .

[5] S.Y.R. Hui and W.C. Ho, A New Generation of Universal Contactless Battery Charging Platform for Portable Consumer Electronic Equipment, 35th Annual IEEE Power Elecrronics Specialists Conference, Aachen, Germany, 2004.

[6] J.J. Casanova, Z.N. Low, J. Lin, and R. Tseng, Transmitting Coil Achieving Uniform Magnetic Field Distribution for Planar Wireless Power Transfer System, Proceedings of the 4th international conference on Radio and wireless symposium, San Diego, CA, USA, 2009.

[7] S. H. Schuder, "Energy transport into the closed chest from a set of very-large mutually orthogonal coils," Transactions of the American Institute of Electrical Engineers. Part 1: Communication and electronics. , vol. 64, pp. 527-534, 1963.

[8] D. Kacprzak, "A Novel S-Pickup for High Power Inductive Power Transfer Systems," in IEEE International Magnetics Conference, INTERMAG 2006. p.204, 2006

[9] G.A.J. Elliott, G.A. Covic, D. Kacprzak and J.T. Boys, "A New Concept: Asymmetrical Pick-Ups for Inductively Coupled Power Transfer Monorail Systems", IEEE Transactions on Magnetics, vol. 42, Issue: 10, Page(s): 3389 - 3391, 2006.

[10] Kacprzak, Dariusz, "Implementation of Finite Element Method Modelling Tools in Education Programs," 2006 6th International Conference on Computational Electromagnetics (CEM) , vol., no., pp.1-2, 4-6 April 2006.

[11] D. Kacprzak, M. J. Gustafsson, M. P. Taylor, "A Finite Element Method Approach to the Design Process of an Aluminum Reduction Cell" IEEE International Magnetics Conference, IEEE Transactions on Magnetics, p. 668, 2006.
[12] Tupsie S., Isaramongkolrak A., Paolaor P., Analysis of electromagnetic field effects using FEM for transmission lines transposition World Academy of Science, Engineer-ing and Technology, pp. 870874, 2009.

[13] O. H. Stielau and G. A. Covic, 'Design of loosely coupled inductive power transfer systems," Proceedings of International Conference on Power Systems Technology., vol. 51, pp. 85-90, 2000.

[14] O’Brien K., Teichmann R., Gueldner H., Magnetic Field Generation in an Inductively Coupled RadioFrequency Power Transmission System, 37th IEEE Power Electronics Specialists Conference, Jeju, Korea, pp2-81, 2006. 